\title{
Discrete-Time Resonant Observer Based Control for Periodic Signal Rejection
}

\author{
J. A. Cortés, G. A. Ramos and R. Costa Castelló*
}

\begin{abstract}
Rejection of periodic signals has been a subject of great interest in control theory and engineering applications. Periodic disturbances are usually present in mechatronic, hydraulic and electronics systems, between others. This paper proposes a resonant observer-based control for periodic signal rejection. The observer includes, in an embedded way, an internal model of the disturbance that is based on its harmonic decomposition. The resulting disturbance estimation is used by a control law that cancels the periodic disturbance term while controlling a specified tracking task. The proposed scheme lets the control designer to address the disturbance estimation and tracking problems separately. Experimental results, on a mechatronic test bed, show that the proposed resonant observerbased control successfully rejects periodic disturbances.
\end{abstract}

Keywords - periodic signals, resonant observer, disturbance observer.

\section{INTRODUCCIÓN}

$\mathrm{E}$ rechazo de perturbaciones periódicas es un amplio tema de estudio en sistemas de control dado que este tipo de perturbaciones se presenta de manera natural en muchas aplicaciones. Dentro de estas aplicaciones se encuentran sistemas mecatrónicos [1], convertidores electrónicos [2] y robo tica [3], entre otros.

Las estrategias de control más comúnmente usadas para el rechazo de perturbaciones periódicas son el Control Repetitivo [1], [4] y control basado en resonadores [5]. Alternativamente, este problema puede ser tratado usando un esquema de control basado en un observador. Bajo este enfoque, el observador se encarga de realizar una estimación de la perturbación que es usada por la ley de control para rechazar la perturbación real. Una tendencia muy relacionada con este enfoque es el Control por Rechazo Activo de Perturbaciones (ADRC) [6]. La técnica ADRC usa modelos lineales para aproximar localmente, de manera polinómica, perturbaciones endógenas y exógenas [7], [8]. Recientemente el Control Proporcional Integral Generalizado (GPI), siguiendo la línea de ADRC, se ha aplicado efectivamente en el rechazo de una amplia gama de perturbaciones [9]. Adicionalmente, un esquema de estimación algebraica de la perturbación es propuesto en [10], para el control de un convertidor DC-DC tipo Cuk.

En este trabajo se propone un control basado en un observador para el rechazo de perturbaciones periódicas. El

*J. A. Cortés, Universidad Nacional de Colombia, Bogotá, Colombia, jacortesr@unal.edu.co

G. A. Ramos, Universidad Nacional de Colombia, Bogotá, Colombia, garamosf@unal.edu.co

R. Costa Castelló, Universitat Politècnica de Catalunya, Barcelona, España,ramon.costa@upc.edu observador planteado se formula de tal manera que incluye un modelo interno de una señal periódica. Este modelo interno se construye a partir de la descomposición frecuencial de la señal periódica. De esta manera, el observador tiene la capacidad de estimar los estados de la planta y cada uno de los componentes frecuenciales seleccionados.

En [11] se propone un procedimiento de sintonización en el que los resonadores se ubican en el lazo de control principal. Como se muestra en esa propuesta, se asignan una ganancia y una fase a cada elemento resonador para obtener márgenes de estabilidad apropiados en un marco de diseño frecuencial. Otra opción de sintonización basada en pasividad se presenta en [12] y [13]. Sin embargo, en ninguno de estos casos la estabilidad del sistema es garantizada por construcción y se requieren de filtros adicionales para un correcto planteamiento del sistema de control. En [14] se realiza un análisis de la respuesta en el tiempo pero no se tiene un manejo adecuado de la respuesta transitoria. Para los dos casos anteriores se utiliza una formulación entrada-salida, no se obtienen directamente una estimación de la perturbación y en general el diseño resultante es limitado.

La validación experimental de la propuesta se realiza en una plataforma mecatrónica giratoria basado en un motor de corriente continua sujeto a un par de perturbación periódico. Los resultados experimentales demuestran que el enfoque propuesto obtiene un desempeño comparable al obtenido con el control repetitivo y el control basado en resonadores, sin embargo, el método de sintonización aquí presentado resulta más simple y directo. El esquema de control propuesto está basado en una formulación en espacio de estado y garantiza por construcción la estabilidad del sistema. A diferencia de los esquemas de control clásicos para el manejo de señales periódicas, control repetitivo y control resonante, la arquitectura propuesta ofrece la ventaja de diseñar independientemente el rechazo de perturbaciones y seguimiento de señales de referencia. Es posible plantear diferentes metodologías de sintonización, entre las más utilizadas: ubicación de polos y control óptimo. En este trabajo se propone una metodología combinada en la cual se usa ubicación de polos para el seguimiento de referencia y el enfoque óptimo de Kalman-Bucy para la configuración del observador resonador en el rechazo de la perturbación. El esquema propuesto en este trabajo usa un procedimiento sencillo de sintonización de las ganancias del observador basado en el filtro de Kalman-Bucy. Este procedimiento ubica de manera óptima los valores propios del sistema observador mediante el ajuste de un único pará metro. Finalmente, por medio de la respuesta en frecuencia del observador se puede verificar el desempeño en estado estacionario del estimador de 
perturbación.

Este artículo está organizado de la siguiente forma: la Sección II describe la arquitectura del controlador propuesto, la Sección III describe la plataforma y los resultados experimentales, finalmente las conclusiones y trabajo futuro se plantean en la Sección IV.

\section{ESTRUCTURA DEL CONTROL BASADO EN OBSERVADOR RESONANTE}

En este trabajo se propone un esquema de control basado en un observador el cual tiene la capacidad de las estimar perturbaciones $T_{p}$-periódicas presentes en el sistema. Se propone construir un modelo extendido del sistema por medio de un modelo de la perturbación. El diseño del observador se realiza sobre la base de este modelo extendido y se encarga de estimar los estados de la planta y la perturbación periódica equivalente asociada. La estructura del sistema de control esta motivada por la filosofía del Control por Rechazo Activo de Perturbaciones (ADRC) pero en este trabajo se considera un modelo diferente de la perturbación que es especializado para señales periódicas. La ley de control lineal propuesta usa el estimado de esta perturbación equivalente para realizar su correspondiente rechazo.

Considere el siguiente modelo de estado lineal y discreto de la planta:

$$
\begin{aligned}
& \mathbf{x}_{k+1}^{p}=\mathbf{A}^{p} \mathbf{x}_{k}^{p}+\mathbf{B}^{p} u_{k}+\mathbf{B}^{p} \xi_{k} \\
& y_{k}=\mathbf{C}^{p} \mathbf{x}_{k}^{p}
\end{aligned}
$$

donde $\mathbf{x}_{k}^{p} \in \mathbf{R}^{n}$ es el vector de estado, $u_{k} \in \mathbf{R}$ es la acción de control, $\xi_{k} \in \mathbf{R}$ es la señal de perturbación y $y_{k} \in \mathbf{R}$ es la salida del sistema. Similarmente, $\mathbf{A}^{p} \in \mathbf{R}^{n \times n}$ es la matriz de transición de estados, $\mathbf{B}^{p} \in \mathbf{R}^{n \times 1}$ es el vector de entrada $\mathrm{y}$ $\mathbf{C}^{p} \in \mathbf{R}^{1 \times n}$ es el vector de salida. El sistema definido por $\left(\mathbf{A}^{\mathrm{p}}, \mathbf{B}^{\mathrm{p}}, \mathbf{C}^{\mathrm{p}}, \mathbf{0}\right)$ se asume como una representación mínima de un sistema observable y controlable. La planta se asume conocida y la perturbación $\xi_{k}$ se asume $N$-periódica (i.e. $\xi_{k}=\xi_{k-N}$ ) desconocida pero acotada, donde $N \in \mathrm{N}$ corresponde al periodo.

Nótese que en las ecuaciones y la perturbación $\xi_{k}$ puede ser considerada como adicionada a la señal de control $u_{k}$. Sin embargo, esta estrategia es aplicable para el rechazo de perturbaciones que entran al sistema por otros canales dado el concepto de la perturbación equivalente a la entrada (ver [15]). Se define la perturbación equivalente a la entrada como aquella señal que, entrando por el canal de la señal de control, ocasiona el mismo efecto sobre la salida del sistema que las perturbaciones originales entrando por otros canales. Por lo tanto, la perturbación estimada por el observador será siempre la perturbación equivalente a la entrada.

\section{A. Modelo de la perturbación}

Dado el carácter $N$-periódico de la señal de perturbación, Ésta puede ser escrita como la suma finita de componentes sinusoidales:

$\xi_{k}=\xi_{k}^{1}+\xi_{k}^{2}+\cdots+\xi_{k}^{m}$

donde

$\xi_{k}^{l}=g^{l} \sin \left(\theta^{l} k+\varphi^{l}\right)$

Con $g^{l}$ la amplitud, $\varphi^{l}$ la fase y $\theta^{l}$ la frecuencia normalizada. Los componentes pueden ser seleccionados de acuerdo al contenido armónico, e. g.: $\quad \theta^{l}=\frac{2 \pi}{N} l \quad \mathrm{rad}, \quad$ siendo $l=0, \ldots, m$ y $\theta^{l}$ la frecuencia fundamental.

Cada término senoidal puede generarse por el siguiente sistema lineal, con condiciones iniciales apropiadas:

$\mathbf{z}_{k+1}^{l}=\mathbf{A}^{l} \mathbf{z}_{k}^{l}$

$\xi_{k}^{l}=\mathbf{C}^{l} \mathbf{z}_{k}^{l}$

$\operatorname{con} \mathbf{z}_{k}^{l} \in \mathbf{R}^{2}, \mathbf{A}^{l}=\left[\begin{array}{cc}0 & 1 \\ -1 & 2 \cos \theta^{l}\end{array}\right]$ y $\mathbf{C}_{k}^{l}=[1,0]$.

Por lo tanto, la señal de perturbación, $\xi_{k}$, admite la siguiente representación en espacio de estado:

$$
\begin{aligned}
& \mathbf{z}_{k+1}=\mathbf{A}^{\xi} \mathbf{z}_{k} \\
& \xi_{k}=\mathbf{C}^{\xi} \mathbf{z}_{k}
\end{aligned}
$$

donde $\mathbf{z}_{k}=\left[\begin{array}{llll}\left(\mathbf{z}_{k}^{1}\right)^{T} & \left(\mathbf{z}_{k}^{2}\right)^{T} & \cdots & \left(\mathbf{z}_{m}^{1}\right)^{T}\end{array}\right]^{T} \in \mathbf{R}^{2 m} \mathrm{y}$

$$
\begin{aligned}
\mathbf{A}^{\xi} & =\left[\begin{array}{ccccc}
\mathbf{A}^{1} & 0 & 0 & \cdots & 0 \\
0 & \mathbf{A}^{2} & 0 & \cdots & 0 \\
0 & 0 & \mathbf{A}^{2} & \cdots & 0 \\
\vdots & \vdots & \vdots & \ddots & \vdots \\
0 & 0 & 0 & \cdots & \mathbf{A}^{m}
\end{array}\right], \\
\mathbf{C}^{\xi} & =\left[\begin{array}{lllll}
\mathbf{C}^{1} & \mathbf{C}^{2} & \mathbf{C}^{3} & \cdots & \mathbf{C}^{m}
\end{array}\right] .
\end{aligned}
$$

este tipo de modelo interno puede ser fácilmente extendido a señales compuestas por múltiples frecuencias o sistemas con distribución de frecuencias arbitrarias.

\section{B. Sistema aumentado}

Se puede construir un modelo aumentado del sistema adicionando al modelo de la planta el modelo obtenido de la señal de perturbación, con lo que se obtiene:

$$
\begin{aligned}
\mathbf{x}_{k+1} & =\mathbf{A} \mathbf{x}_{k}+\mathbf{B} u_{k} \\
y_{k} & =\mathbf{C} \mathbf{x}_{k}
\end{aligned}
$$

donde:

$$
\begin{aligned}
& \mathbf{x}_{k}=\left[\begin{array}{ll}
\left(\mathbf{x}_{k}^{p}\right)^{T} & \left(\mathbf{z}_{k}\right)^{T}
\end{array}\right] \in \mathbf{R}^{n+2 m}, \mathbf{A}=\left[\begin{array}{cc}
\mathbf{A}^{p} & \mathbf{B}^{p} \mathbf{C}^{\xi} \\
\mathbf{0} & \mathbf{A}^{\xi}
\end{array}\right] \in \mathbf{R}^{(n+2 m) \times(n+2 m)}, \\
& \mathbf{B}=\left[\begin{array}{c}
\mathbf{B}^{p} \\
\mathbf{0}
\end{array}\right] \in \mathbf{R}^{n+2 m}, \quad \mathbf{C}=\left[\begin{array}{ll}
\mathbf{C}^{\mathbf{p}} & \mathbf{0}
\end{array}\right] \in \mathbf{R}^{1 \times(n+2 m)} .
\end{aligned}
$$

Este sistema, con condiciones iniciales apropiadas, es equivalente a (1)-(2) sujeto a (3)-(4). Es importante notar que (7)-(8) no tiene entrada de perturbación y es un sistema observable pero no es un sistema completamente controlable. El subsistema controlable corresponde al modelo del planta y el subsistema no controlable corresponde a el modelo de la 
perturbación. Nótese que la perturbación es una señal exógena y no puede ser modificada por medio de la acción de control.

\section{Observador resonante}

En esta sección se propone un observador lineal estilo Luenberger para la estimación del estado del sistema aumentado (7)-(8):

$$
\mathbf{x}_{k+1}=\mathbf{A} \hat{\mathbf{x}}_{k}+\mathbf{B} u_{k}+\mathbf{L}\left[y_{k}-\mathbf{C} \hat{\mathbf{x}}_{k}\right] \text {, }
$$

donde $\quad \mathbf{x}_{k}=\left[\begin{array}{ll}\left(\hat{\mathbf{x}}_{k}^{p}\right)^{T} & \hat{\mathbf{z}}_{k}^{T}\end{array}\right]^{T}$ es el vector de estados estimados del sistema aumentado y $\mathbf{L}=\left[\begin{array}{ll}\left(\mathbf{L}^{p}\right)^{T} & \left(\mathbf{L}^{\xi}\right)^{T}\end{array}\right]^{T}$ son las ganancias del observador.

El observador (9) también puede ser representado por el siguiente sistema en lazo cerrado:

$$
\mathbf{x}_{k+1}=(\mathbf{A}-\mathbf{L C}) \hat{\mathbf{x}}_{k}+\mathbf{B} u_{k}+\mathbf{L} y_{k} \text {. }
$$

Nótese que $\mathbf{Z}_{k}$ es compuesto por $\left[\left(\mathbf{z}_{k}^{1}\right)^{T}, \cdots,\left(\hat{\mathbf{z}}_{k}^{l}\right)^{T}, \cdots,\left(\hat{\mathbf{z}}_{k}^{m}\right)^{T}\right]^{T}$ y el primer elemento de $\mathbf{z}_{k}^{\prime}$, corresponde a la estimación de $\xi_{k}^{l}$. Por lo tanto la estimación de la perturbación es:

$\hat{\xi}_{k}=\mathbf{C}^{\xi} \hat{\mathbf{z}}_{k}$.

$$
\text { Definiendo } \quad \mathbf{e}_{k}=\mathbf{x}_{k}-\hat{\mathbf{x}}_{k}=\left[\left(\mathbf{e}_{k}^{p}\right)^{T},\left(\mathbf{e}_{k}^{\xi}\right)^{T}\right]^{T}
$$

sustrayendo el sistema observador (10) del modelo (7) se obtiene:

$$
\mathbf{e}_{k+1}=(\mathbf{A}-\mathbf{L C}) \mathbf{e}_{k}
$$

que describe la dinámica del error de estimación. El polinomio característico es definido como:

$$
\operatorname{det}(\boldsymbol{Z}-\mathbf{A}+\mathbf{L C}) \text {. }
$$

Los valores propios del sistema observador pueden ser arbitrariamente ubicados por la selección adecuada del vector de ganancias $\mathbf{L}$.

\section{Control basado en un observador resonante}

En esta sección se definirá la acción de control que será empleada para rechazar las perturbaciones periódicas del sistema. De esta manera, un controlador por realimentación de estado para la planta (1)-(2) puede ser aplicado usando la estimación obtenida por medio del observador (10):

$$
u_{k}=-\mathbf{K}^{p} \hat{\mathbf{x}}_{k}^{p}-\mathbf{C}^{\xi} \hat{\mathbf{z}}_{k}
$$

Donde $\mathbf{K}^{p}$ corresponde con el vector de ganancias de realimentación de estado. La ley de control logra el rechazo de la perturbación por medio del término $\mathbf{C}^{\xi} \hat{\mathbf{z}}_{k}$, ver (11).

Este controlador realiza el rechazo de perturbaciones y regula el estado del sistema hacia el origen, sin embargo, dado que la mayoría de aplicaciones requieren tareas de seguimiento, esta ley de control se complementa incluyendo el modelo interno de la señal de referencia: $\mathbf{x}_{k+1}^{i m}=\mathbf{A}^{i m} \mathbf{x}_{k}^{i m}+\mathbf{B}^{i m}\left(r_{k}-y_{k}\right)$,

Donde $\mathbf{A}^{\text {im }}$ corresponde con la matriz de transición de estado y $\mathbf{B}^{i m}$ es el vector de entrada del modelo interno.

Observación: Para el caso de referencias seccionalmente constantes el modelo interno corresponde a un integrador. Como consecuencia $\mathbf{A}^{i m}=1$ y $\mathbf{B}^{i m}=1$.

Tomando en consideración el modelo interno, la ley de control completa toma la siguiente forma:

$u_{k}=\mathbf{K}^{i m} \mathbf{x}_{k}^{i m}-\mathbf{K}^{p} \hat{\mathbf{x}}_{k}^{p}-\mathbf{C}^{\xi} \hat{\mathbf{z}}_{k}$,

Donde $\mathbf{K}^{\text {im }}$ es la ganancia de realimentación del modelo interno.

Con este controlador se obtiene la siguiente matriz de transición de estado del sistema general en lazo cerrado:

$\mathbf{A}^{c l}=\left[\begin{array}{cc}\mathbf{A}^{a}-\mathbf{B}^{a} \mathbf{K}^{a} & -\mathbf{B}^{a} \mathbf{K} \\ \mathbf{0} & \mathbf{A}-\mathbf{L} \mathbf{C}\end{array}\right]$,

con $\left[\left(\mathbf{X}_{k}^{p}\right)^{T},\left(\mathbf{X}_{k}^{i m}\right)^{T},\left(\mathbf{e}_{k}^{p}\right)^{T},\left(\mathbf{e}_{k}^{\xi}\right)^{T}\right]^{T}$ como vector de estado y $\mathbf{A}^{a}=\left[\begin{array}{cc}\mathbf{A}^{p} & \mathbf{0} \\ -\mathbf{B}^{i m} \mathbf{C}^{p} & \mathbf{A}^{i m}\end{array}\right], \mathbf{B}^{a}=\left[\begin{array}{c}\mathbf{B}^{p} \\ \mathbf{0}\end{array}\right], \mathbf{K}^{a}=\left[\begin{array}{cc}\mathbf{K}^{p} & -\mathbf{K}^{i m}\end{array}\right]$, $\mathbf{K}=\left[\begin{array}{ll}\mathbf{K}^{p} & \mathbf{C}^{\xi}\end{array}\right]$

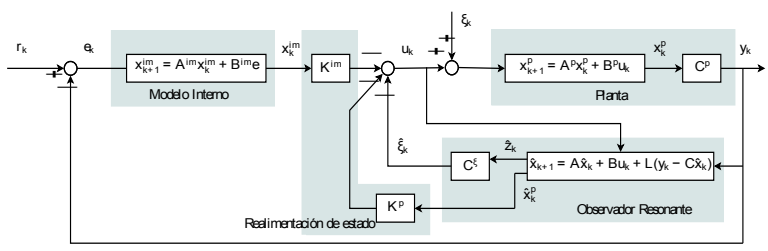

Figura 1. Estructura del control basado en un observador resonante.

La dinámica en lazo cerrado puede ser determinada ajustando los valores propios de la matriz de transición de estado. Esta tarea puede ser realizada mediante la selección de $\mathbf{K}^{p}, \mathbf{K}^{i m}, \mathbf{L}^{p}$ y $\mathbf{L}^{\xi}$. De la matriz de transición y basado en el principio de separación [16] se puede diseñar de manera independiente los vectores de ganancias del controlador $\mathbf{K}^{p}$, $\mathbf{K}^{i m}$, de los vectores de ganancias de estimación $\mathbf{L}^{p}$ y $\mathbf{L}^{\xi}$.

\section{E. Sintonización}

La arquitectura propuesta incluye el modelo interno de la referencia y el modelo interno de la perturbación. Por lo tanto, bajo el principio del modelo interno, esto garantiza que el error de seguimiento en estado estacionario es nulo si el sistema es estable. Como consecuencia el desempeño obtenido en estado estacionario será similar al obtenido usando control repetitivo o control resonante. A diferencia de estas estrategias la arquitectura propuesta garantiza estabilidad en lazo cerrado por construcción. Los parámetros de diseño de la arquitectura son $\mathbf{K}^{a}$ y $\mathbf{L}$ los cuales pueden ser seleccionados de diferentes maneras:

- Ubicación de polos de lazo cerrado. Los componentes más relevantes de la respuesta en el tiempo (tiempo de convergencia, frecuencia de oscilación, etc.) pueden ser completamente especificados fijando los valores propios de (16). 
- Diseño óptimo. Usualmente los problemas de control y estimación pueden ser formulados como la solución de un problema de optimización [17]. Esto incluye Regulador Lineal Cuadrático (LQR) y filtro de Kalman.

- Métodos combinados. Dada la estructura de los parámetros pueden diseñarse de manera desacoplada. Esto permite dar un tratamiento diferente para el seguimiento de referencias y el rechazo de perturbaciones.

En este trabajo se usa la tercera opción y se propone usar el filtro de Kalman-Bucy como método de selección de las ganancias del observador. Mediante este procedimiento se obtiene un diseño óptimo del observador de perturbaciones propuesto en (9) donde el objetivo es minimizar la covarianza del error de estimación (ver por ejemplo [17] para la descripción del método). Por lo tanto el vector de ganancias Óptimo del observador se define como:

\section{$\mathbf{L}=\mathbf{P C}^{T} \mathbf{V}^{-1}$}

donde $\mathbf{P}$ es la solución única positiva semi-definida de la ecuación algebraica de Riccati:

\section{$\mathbf{P} \mathbf{A}^{T}+\mathbf{A P}-\mathbf{P C}^{T} \mathbf{V}^{-1} \mathbf{C P}+\mathbf{W}=\mathbf{0}$,}

Siendo $\mathbf{V}$ y $\mathbf{W}$ las matrices de densidad espectral del ruido de medición y del proceso respectivamente.

Se han seleccionado $\mathbf{V}=1$ y $\quad \mathbf{W}=\alpha[0 \mathbf{C z}]^{T}[0 \mathbf{C z}]$ donde $\alpha$ es una factor de ponderación entre el ruido del sistema y el ancho de banda del observador. Luego $\alpha$ constituye el único parámetro de diseño usado para sintonizar el observador.

Para verificar la correcta sintonización del observador se puede considerar la respuesta en frecuencia del siguiente sistema

$\left[\begin{array}{c}\mathbf{x}_{k+1}^{p} \\ \mathbf{x}_{k+1}^{i m} \\ \hat{\mathbf{x}}_{k+1}^{p} \\ \hat{\mathbf{z}}_{k+1}^{p}\end{array}\right]=\left[\begin{array}{cccc}\mathbf{A}^{p} & -\mathbf{B}^{p} \mathbf{K}^{i m} & -\mathbf{B}^{p} \mathbf{K}^{p} & -\mathbf{B}^{p} \mathbf{C}^{\xi} \\ -\mathbf{B}^{i m} \mathbf{C}^{p} & \mathbf{A}^{i m} & \mathbf{0} & \mathbf{0} \\ \mathbf{L}^{p} \mathbf{C}^{p} & -\mathbf{B}^{p} \mathbf{K}^{i m} & \mathbf{A}^{p}-\mathbf{B}^{p} \mathbf{K}^{p}-\mathbf{L}^{p} \mathbf{C}^{p} & \mathbf{0} \\ \mathbf{L}^{\xi} \mathbf{C}^{p} & \mathbf{0} & -\mathbf{L}^{\xi} \mathbf{C}^{p} & \mathbf{A}^{\xi}\end{array}\right]\left[\begin{array}{c}\mathbf{x}_{k}^{p} \\ \mathbf{x}_{k}^{i m} \\ \hat{\mathbf{x}}_{k}^{p} \\ \hat{\mathbf{z}}_{k}^{p}\end{array}\right]+\left[\begin{array}{c}\mathbf{B}^{p} \\ \mathbf{0} \\ \mathbf{0} \\ \mathbf{0}\end{array}\right] \xi_{k}$

$\left[\begin{array}{l}\hat{\xi}_{k} \\ y_{k}\end{array}\right]=\left[\begin{array}{cccc}\mathbf{0} & \mathbf{0} & \mathbf{0} & \mathbf{C}^{\xi} \\ \mathbf{C}^{p} & \mathbf{0} & \mathbf{0} & \mathbf{0}\end{array}\right]\left[\begin{array}{c}\mathbf{x}_{k}^{p} \\ \mathbf{x}_{k}^{i m} \\ \hat{\mathbf{x}}_{k}^{p} \\ \hat{\mathbf{z}}_{k}\end{array}\right]$

El sistema (17) es el sistema en lazo cerrado con entrada la perturbación real $\xi$ y salidas $\hat{\xi}_{k}$ y $y_{k}$. Al graficar la respuesta en frecuencia para la salida $\hat{\xi}_{k}$ se comprueba el desempeño en cuanto a la estimación de la perturbación.

\section{PlatAFORMA EXPERIMENTAL}

A. Descripción de la planta

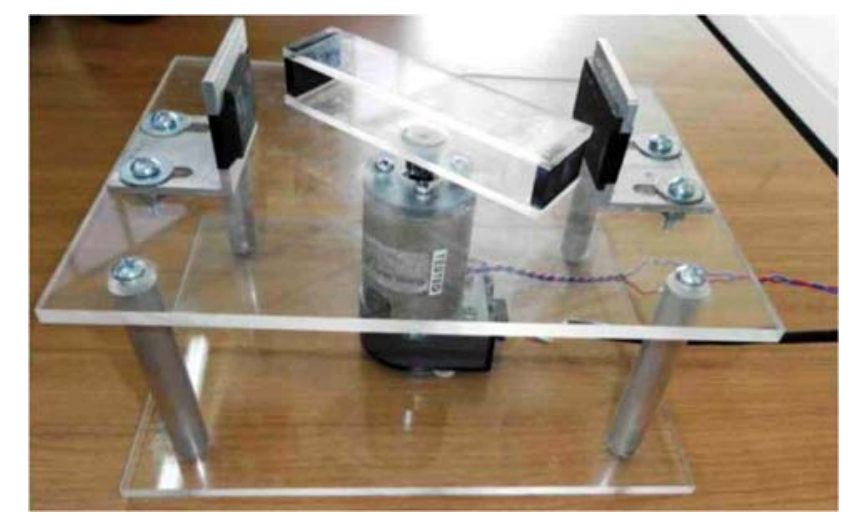

Figura 2. Fotografía de la plataforma experimental: motor DC, encoder óptico, sistema magnético (carga), y estructura de soporte.

La Fig. 2 muestra la planta experimental (para una descripción detallada de este sistema ver [18]). La planta está compuesta por una barra que sostiene un imán permanente en cada extremo en orientación magnética opuesta. La barra está anclada al eje de un motor dc y al girar queda expuesta al efecto de dos electro-imanes fijos dispuestos sobre la estructura del sistema. La rotación del motor dc causa un par de carga pulsante $\left(\boldsymbol{\Gamma}_{p}\right)$ que depende de la posición angular del eje del motor. Cuando la velocidad angular del eje, $\omega$, es constante, i.e. $d \omega / d t=0$, el par de carga es una señal periódica cuyo periodo fundamental está directamente relacionado con esta velocidad: $\mathrm{T}_{\mathrm{p}}=\omega^{-1}$, con $\omega$ expresado en rev/s. El objetivo de control es mantener constante la velocidad de giro del motor a un valor deseado. El motor dc es operado mediante un amplificador de transconductancia, por lo tanto la acción de control puede ser considerada como el par aplicado al eje del motor. Bajo estas consideraciones el modelo del sistema puede escribirse como:

$J \dot{\omega}=-B \omega+\Gamma_{p}+\Gamma$

Donde $\boldsymbol{\Gamma}$ corresponde al par de control aplicado por el motor dc. Este modelo puede escribirse en forma de entrada/salida:

$G_{p}(s)=\frac{K}{\tau s+1}=\frac{31}{0.62 s+1} \frac{\mathrm{rev} / \mathrm{s}}{\mathrm{V}}$.

Cabe notar que en este caso, la entrada corresponde al voltaje de referencia aplicado al amplificador. Finalmente, este modelo de tiempo continuo puede ser reformulado en tiempo discreto usando un periodo de muestreo $T_{S}=1 \mathrm{~ms}$ :

$G_{p}(z)=G_{p}\left(z, T_{s}\right)=\frac{K\left(1-e^{-T_{s} / \tau}\right)}{z-e^{-T_{s} / \tau}}=\frac{0.001431}{z-0.9984}$.

\section{B. Resultados experimentales}

La Fig. 3 muestra la respuesta del sistema en lazo abierto a velocidad nominal. Puede observarse como el par de perturbación afecta a la velocidad. Adicionalmente se presenta el contenido armónico de la señal de salida obtenida.

El controlador propuesto se diseña a partir del modelo (19), para una velocidad nominal de $\omega=4 \mathrm{rev} / \mathrm{s}$ y con el periodo de muestreo seleccionado se obtienen 250 muestras por periodo, esto es $N=250$. El controlador se diseña con el ajuste $\mathbf{K}^{i m}$ y $\mathbf{K}^{p}$. Estos parámetros han sido definidos para fijar todos los 
polos de lazo cerrado, valores propios de $\left(\mathbf{A}^{a}-\mathbf{B}^{a} \mathbf{K}^{a}\right)$, en 0.94, correspondiente a una respuesta no oscilatoria con constante de tiempo de $16.16 \mathrm{~ms}$.

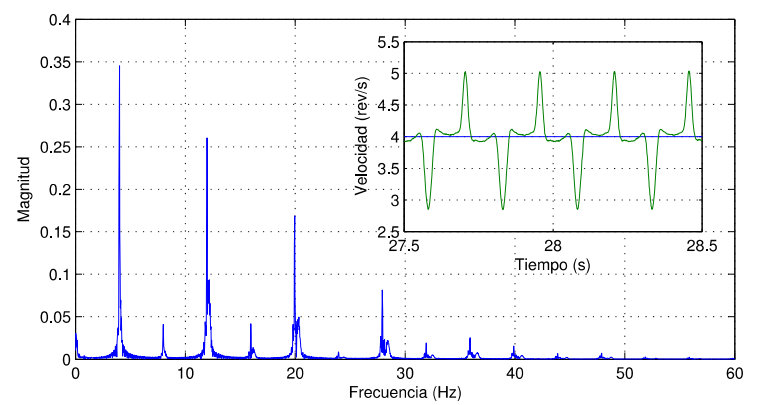

Figura 3. Velocidad de salida en lazo abierto y su contenido armónico.

Para esta aplicación se asume que la perturbación puede aproximarse suficientemente usando 15 componentes armónicos (ver Fig. 3). Teniendo esto en cuenta, se utiliza el enfoque del filtro de Kalman para ajustar las ganancias del estimador de perturbación, $\mathbf{L}$.

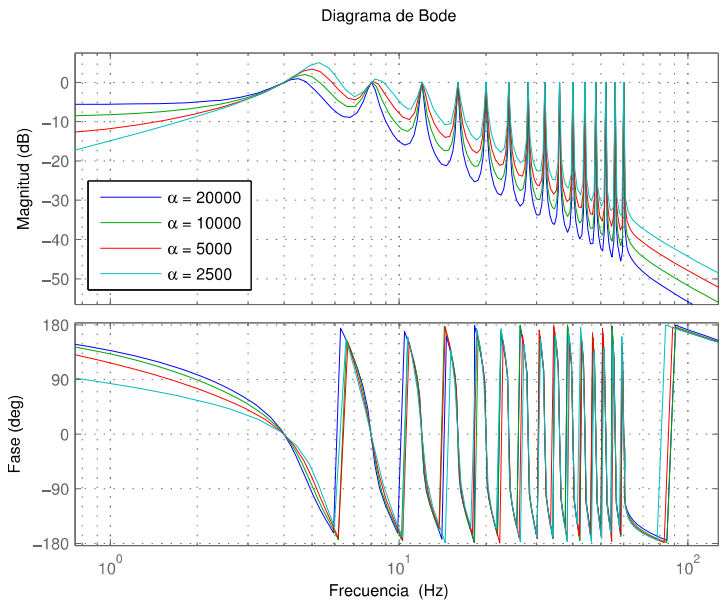

Figura 4. Respuesta en frecuencia de $\xi(z) / \hat{\xi}(z)$ para diferentes valores de $\alpha$.

La respuesta en frecuencia del sistema (17) se muestra en las Figuras 4 y 5 para diferentes valores de $\alpha$. En la Fig. 4 puede verse que para la frecuencia fundamental de $4 \mathrm{~Hz}$ y los 14 armónicos siguientes se tiene una ganancia igual a $1(0 \mathrm{~dB}$ en la gráfica de magnitud y $0^{\circ}$ en la gráfica de fase). Esto significa que los componentes de la perturbación $\xi_{k}$ con estas frecuencias se verán directamente reflejados en $\hat{\xi}_{k}$. Puede observarse que para el conjunto de valores de $\alpha$ seleccionados, con $\alpha=2500$ se presenta la mayor ganancia para las frecuencias inter-armónicas. Esto significa un mayor desempeño, sin embargo, si estas ganancias son muy grandes se obtiene un sistema con alta sensibilidad al ruido. La Fig. 5 muestra la respuesta en frecuencia del rechazo de la perturbación. Se puede observar una alta atenuación para los componentes armónicos considerados en el diseño del observador resonador (menor a $160 \mathrm{~dB}$ ). Para $\alpha=2500$ se presenta la mayor atenuación para la frecuencias interarmónicas.

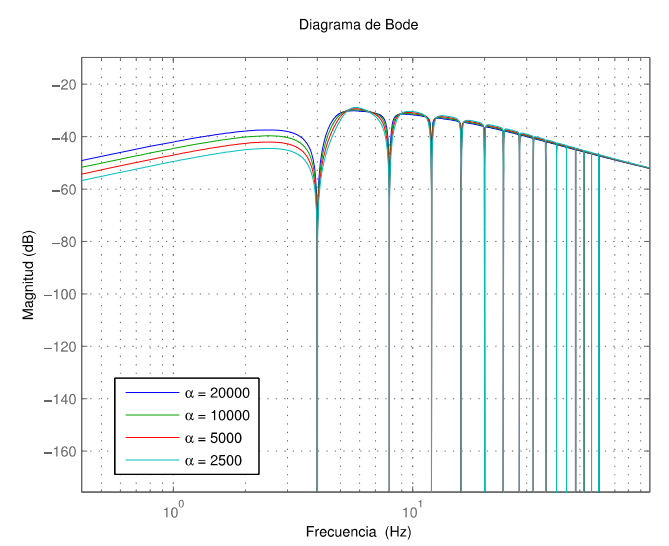

Figura 5. Velocidad Respuesta en frecuencia de $Y(z) / \xi(z)$.

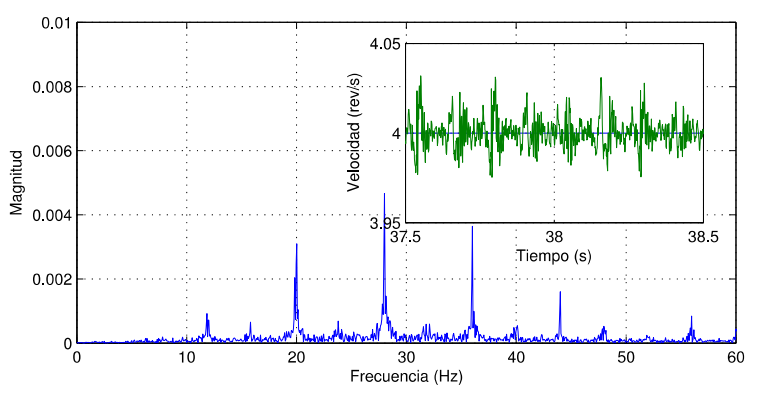

Figura 6. Velocidad Respuesta en el tiempo de lazo cerrado con $\alpha=20000$ y su contenido armónico.

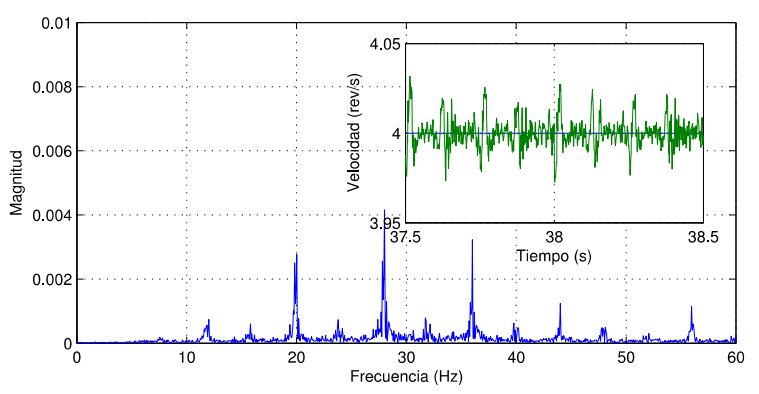

Figura 7. Velocidad Respuesta en el tiempo de lazo cerrado con $\alpha=10000$ y su contenido armónico.

Las Figuras 6, 7 y 8 presentan el comportamiento en estado estacionario y su correspondiente respuesta en frecuencia para los valores de $\alpha$ de 20000, 10000 y 2500 respectivamente. Se puede apreciar la atenuación de los componentes armónicos en cada caso, resultando para 2500 la mayor atenuación obtenida. Este resultado se corrobora con las correspondientes respuestas en el dominio del tiempo en estado estacionario, en donde se percibe un mejor desempeño para 2500. 


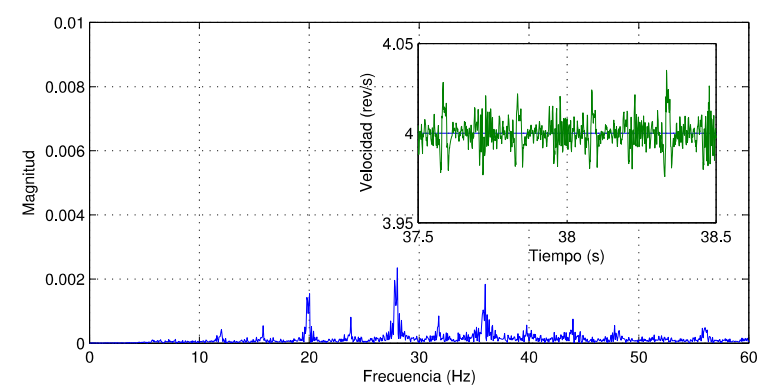

Figura 8. Velocidad Respuesta en el tiempo de lazo cerrado con $\alpha=2500$ y su contenido armónico.

En la Fig. 9 se presenta la respuesta transitoria para diferentes valores de $\alpha$. El tiempo de asentamiento para todos los casos esta alrededor de $5 \mathrm{~s}$. Puede observarse que el comportamiento transitorio mejora a medida que $\alpha$ disminuye, sin embargo, para valores de $\alpha$ menores de 2500 comienza a presentarse una mayor sensibilidad al ruido.
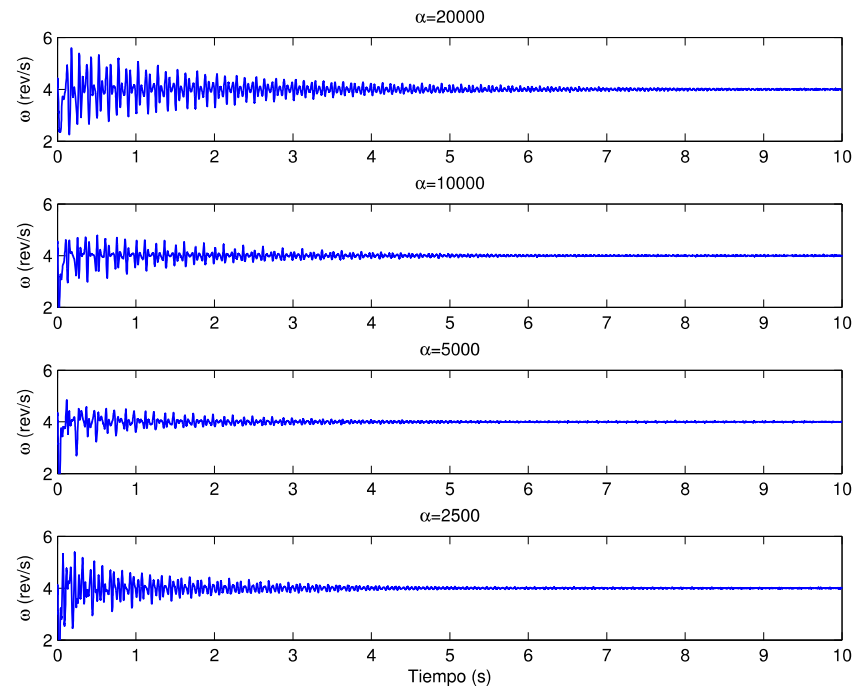

Figura 9. Respuesta transitoria para diferentes valores de $\alpha$.

El diseño propuesto exhibe robustez frente a la variación de los parámetros de la planta. Los cambios en la dinámica por la variación de los parámetros pueden verse, de manera equivalente, como una entrada de perturbación que es parcialmente estimada por el observador. Se realizó un experimento enfocado a determinar la sensibilidad del sistema de control a la variación de los parámetros de la planta. Para tal fin, se rediseña el observador ( $\operatorname{con} \alpha=2500$ ) suponiendo unos parámetros modificados $(\bar{\tau}, \bar{K})$ de la planta continua en un $25 \%$ así: $\bar{\tau}=1.25 \tau$ y $\bar{K}=0.75 K$. La respuesta en el tiempo del sistema para cada caso se muestra en la Fig. 10. Para el caso de la variación en la constante de tiempo $\tau$ se observa un desempeño comparable con el obtenido para el caso de los parámetros reales de la planta (ver Figura 9). Con respecto a la variación de la constante proporcional del sistema $K$, la respuesta exhibe un notable aumento del tiempo de asentamiento ( 9 s) que es debido a la disminución de la ganancia del sistema de control, sin embargo, se realiza un adecuado rechazo de la perturbación equivalente ante la variación del parámetro.
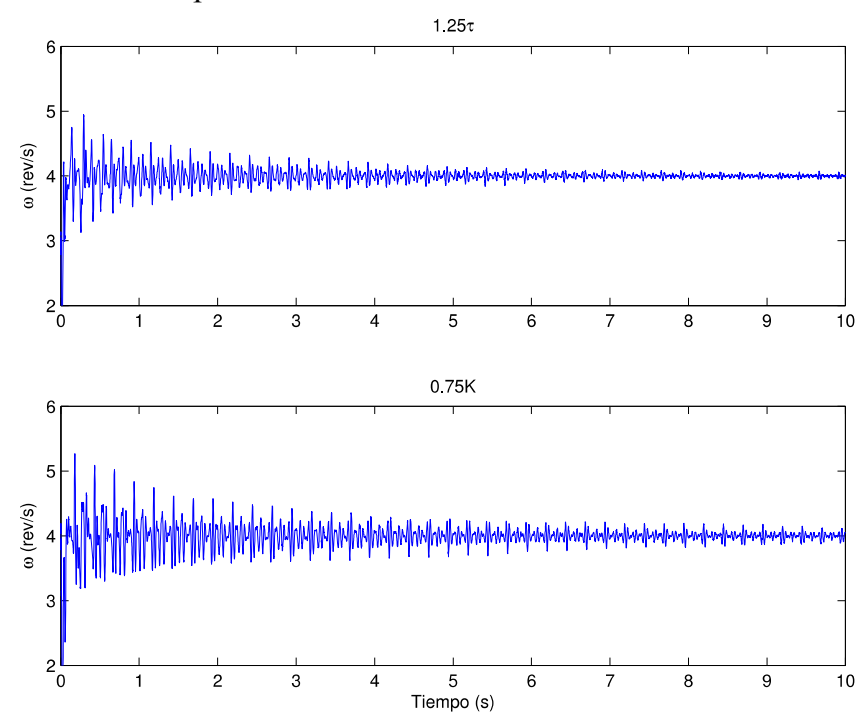

Figura 10. Respuesta en el tiempo para $1.25 \tau$ y $0.75 \mathrm{~K}$.

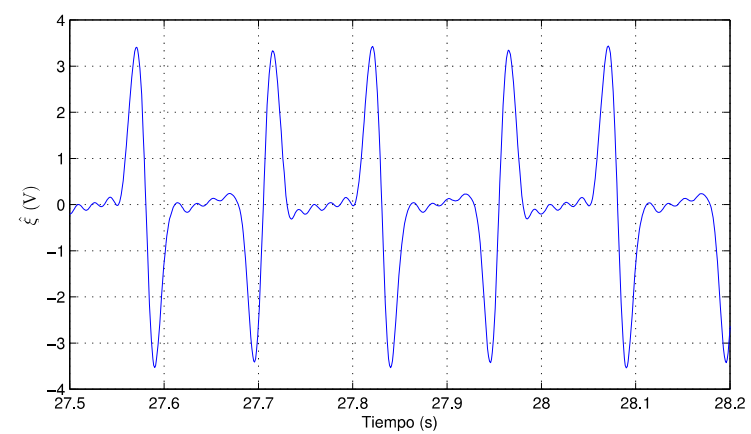

Figura 11. Estimación de la perturbación en estado estacionario.

Finalmente, la salida del observador de perturbación, $\hat{\xi}_{k}$, en estado estacionario se muestra en la Fig. 11. Se puede observar que la señal generada puede ser directamente relacionada con el par pulsante de carga producido por la interacción de los imanes fijos y los imanes móviles.

\section{CONCLUSIONES}

En este trabajo se propuso una nueva arquitectura de control basado en un observador resonante para la estimación y rechazo de perturbaciones periódicas. El observador propuesto estima la descomposición armónica de la perturbación y el controlador usa esta estimación para cancelar su efecto sobre el sistema. El controlador adicionalmente esta diseñado para cumplir tareas de seguimiento. Gracias al principio de separación se puede independizar el diseño del seguimiento del diseño de la estimación de la perturbación. La sintonización del observador resonante se realiza mediante la aplicación del filtro Kalman-Bucy. Esto permite que el desempeño de la estimación se pueda ajustar mediante un único parámetro. Los resultados experimentales muestran que la estrategia propuesta logra efectivamente rechazar la perturbación periódica imponiendo una dinámica adecuada del 
error de seguimiento. Adicionalmente es robusta frente al variación de los parámetros de la planta.

\section{REFERENCIAS}

[1] M. Tomizuka, "Dealing with periodic disturbances in controls of mechanical systems," Annual Reviews in Control, vol. 32, no. 2, pp. 193 $-199,2008$.

[2] G. A. Ramos and R. Costa-Castelló, "Power factor correction and harmonic compensation using second-order odd-harmonic repetitive control," IET Control Theory and Applications, vol. 6, no. 1, pp. 1-12, 2012.

[3] J. Kasac, B. Novakovic, D. Majetic, and D. Brezak, "Passive finitedimensional repetitive control of robot manipulators," Control Systems Technology, IEEE Transactions on, vol. 16, no. 3, pp. 570-576, May 2008.

[4] R. W. Longman, "Iterative learning control and repetitive control for engineering practice," International Journal of Control, vol. 73, no. 10, pp. 930-954, 2000.

[5] Z. Shuai, A. Luo, C. Tu, and D. Liu, "New control method of injectiontype hybrid active power filter," IET Power Electronics, vol. 4, pp. 1051-1057(6), November 2011.

[6] J. Han, "From PID to active disturbance rejection control," IEEE Transactions on Industrial Electronics, vol. 56, no. 3, pp. $900-906$, March 2009.

[7] Z. Gao, "Active disturbance rejection control: a paradigm shift in feedback control system design," in American Control Conference, 2006, Minneapolis, USA, June 2006, pp. 2399-2405.

[8] [8] Z. Gao, Y. Huang, and J. Han, "An alternative paradigm for control system design," in Proceedings of the 40th IEEE Conference on Decision and Control, 2001., vol. 5, Orlando, USA, 2001, pp. 45784585 .

[9] H. Sira, A. Luviano, and J. Cortés, "Control lineal robusto de sistemas no lineales," RIAII, vol. 8, no. 1, pp. 14-28, 2010. [Online]. Available: http://84.88.0.49/index.php/RIAII/article/view/RIAI.2011.01.04

[10] J. Linares Flores, J. L. B. Avalos, and C. Espinosa, "Passivity-based controller and online algebraic estimation of the load parameter of the dc-to-dc power converter cuk type," Latin America Transactions, IEEE (Revista IEEE America Latina), vol. 9, no. 1, pp. 784-791, March 2011.

[11] M. F. Byl, S. J. Ludwick, and D. L. Trumper, "A loop shaping perspective for tuning controllers with adaptive feedforward cancellation," Precision Engineering, vol. 29, no. 1, pp. 27 - 40, 2005.

[12] W. Messner and M. Bodson, "Design of adaptive feedforward controllers using internal model equivalence," in Proc. of the American Control Conference, 1994, pp. 1819-1623.

[13] R. Costa-Castelló, R. Griñó, and E. Fossas, "Resonant control of a single-phase full-bridge unity power factor boost rectifier," in IEEE International Conference on Control Applications, 2007, pp. 599 - 604.

[14] A. Vidal, "Transient response evaluation of stationary-frame resonant current controllers for grid-connected applications," IET Power Electronics, April 2014. [Online]. Available: http://digitallibrary.theiet.org/content/journals/10.1049/iet-pel.2013.0597

[15] J. hua She, M. Fang, Y. Ohyama, H. Hashimoto, and M. Wu, "Improving disturbance-rejection performance based on an equivalentinput-disturbance approach," IEEE Transactions on Industrial Electronics, vol. 55, no. 1, pp. 380-389, 2008.

[16] C.-T. Chen, Analog and Digital Control System Design. Saunders College Publishing, 1993.

[17] B. D. O. Anderson and J. B. Moore, Optimal control: linear quadratic methods. Upper Saddle River, NJ, USA: Prentice-Hall, Inc., 1990.

[18] R. Costa-Castelló, J. Nebot, and R. Griñó, "Demonstration of the internal model principle by digital repetitive control of an educational laboratory plant," Education, IEEE Transactions on, vol. 48, no. 1, pp. 73-80, Feb 2005.

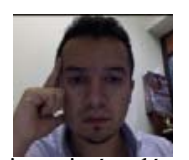

Dr. John Alexander Cortés Romero, recibió los grados de ingeniero electricista, magister en automatización industrial y magister en matemáticas de la Universidad Nacional de Colombia (1995, 1999 y 2007 respectivamente). En el 2011 recibió el grado de doctor en ciencias en la especialidad de ingeniería eléctrica del instituto CINVESTAV de México. Actualmente es profesor asociado adscrito al departamento de ingeniería eléctrica y electrónica de la Universidad Nacional de Colombia sede Bogotá. Sus temas principales de investigación son aplicación de métodos algebraicos en sistemas de control y control por rechazo activo de perturbaciones.
Dr. Germán Andrés Ramos Fuentes es professor asociado adscrito al departamento de ingeniería eléctrica y electrónica de la Universidad Nacional de Colombia. Su investigación está principalmente centrada en el desarrollo de técnicas de control digital para el seguimiento/rechazo de señales periódicas con aplicación a los convertidores de potencia y las plantas mecatrónicas.

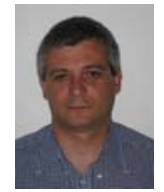

Dr. Ramon Costa Castelló es profesor Titular de Universidad en el departamento de 'Enginyeria de Sistemes Automàtica i Informàtica Industrial" (ESAII) y en el 'Institut d'Organització i Control de Sistemes Industrials" (IOC) de la UPC. Su investigación está principalmente centrada en el desarrollo de técnicas de control digital para el seguimiento/rechazo de señales periódicas con aplicación a los convertidores de potencia y las plantas mecatrónicas. 